\title{
A new selection index percent emphasis method using subindex weights and genetic evaluation accuracy
}

\author{
X. Zhang* $*$ and P. Amer $(1)$ \\ Abacusbio Limited, Dunedin 9016, New Zealand
}

\begin{abstract}
The current international standard methodology to quantify trait percent emphasis in selection indexes is based on a simple multiplication of the relative contribution of each trait's economic value (converted to absolute value) and its genetic standard deviation. This method does not reflect the actual selection emphasis applied when the index is used in practice. The economic value does not reflect selection effort when traits differ considerably in their accuracy of evaluation, and no account is taken for either favorable or antagonistic correlations among traits. A new emphasis method adjusted by both accuracy and genetic correlation is proposed. Genetically highly correlated traits are grouped into subindexes by applying a hierarchical clustering method to the genetic correlation matrix. Then each trait's subindex emphasis is calculated within its subindex group, with a weighting included for trait accuracy. Finally, each subindex emphasis is converted to a full index emphasis according to the conventional relative emphasis of its corresponding subgroup. The method can also be applied to sets of breeding values and their economic weights. When applied to a New Zealand sheep breeding selection index where trait genetic correlations are distinct across subindex groups, the new method shrank the emphasis on low-heritability traits, including survival, from $51 \%$ to $19 \%$; and expanded that on growth traits from $30 \%$ to $49 \%$, better reflecting the selection pressure applied in reality. When genetic correlations across traits were similar, clustering became difficult. Accounting for accuracy affected traits' within-subindex group rankings, whereas the clustering to account for correlations affected all traits within a subgroup equally. Accounting for differences in trait accuracy when describing percent emphasis within selection indexes gives a more practical indication of the likely outcome of selecting on the index. Accounting for correlations among traits when defining percent
\end{abstract}

Received August 30, 2020.

Accepted January 10, 2021.

*Corresponding author: lzhang@abacusbio.co.nz emphasis made a significant difference only in a subset of case study examples.

Key words: selection index, index emphasis, selection response, subindex, index weight

\section{INTRODUCTION}

Selection indexes contain weights given to breeding values for traits under selection. The linear combination of selection index weights and trait estimated breeding values are widely used to rank animals in livestock breeding programs. When explaining selection indexes to farmers, it is helpful to describe and quantify the selection pressure given to each trait. One option for describing selection pressure is to use measures of selection response in each trait, or the reduction in overall economic response if one or more traits were to be removed from the index. Selection response is defined as the change in genetic merit for a trait or index given a certain selection intensity, genetic accuracy, and genetic variation (Falconer and Mackay, 1996). However, it is common to observe unfavorable genetic responses in traits, even when a significant level of selection effort and index pressure is be applied to improve them. One example is the unfavorable genetic response in body size that occurs when selecting for higher milk production. This is due to the positive correlation between milk production traits and mature body size 0.05 to 0.36 (Manzanilla-Pech et al., 2016; William, 2016; VanRaden et al., 2018). Thus, in many selection indexes, mature body size is given a large emphasis in the negative direction to account for the cost of feeding large cows. Only considering selection response in the description of the index obscures the weighting in the index to curb the undesired response in an antagonistic trait that has lower effective weighting than a main selection trait.

Relative weight, also known as the relative emphasis or percent emphasis, has also been used to describe the selection pressure applied to each trait in a way that is comparable to the rest of the traits in a selection index (VanRaden, 2002; Miglior et al., 2005, 2017). This metric is useful to rank the importance of traits and to 
compare across different selection indexes. The current standard methodology to quantify trait emphasis (e) in selection indexes is based on the relative contribution of each trait's economic value, considering a trait's genetic standard deviation (SD) and the total genetic SD of the index (VanRaden, 2002; Miglior et al., 2005, 2017), such as the following:

$$
e_{i}=\frac{\left|a_{i}\right| \sigma_{g_{i}}}{\sum_{i=1}^{t}\left|a_{i}\right| \sigma_{g_{i}}} \times 100 \%,
$$

where $t$ is the total number of traits, and where $e_{i}$, $a_{i}$, and $\sigma_{g i}$ are the selection emphasis, the economic weights, and the SD of the breeding values of the $i$ th trait, respectively; $g$ is the additive genetic effect or breeding value; and $\sigma_{g}$ is the SD of genetic variance. This method does not necessarily reflect the actual selection pressure that occurs in practice and, thus, can be misleading when interpreted by index users who do not have a comprehensive understanding of the index. This is because (1) the economic value is not a direct reflection of practical operation when traits differ considerably in their accuracy of evaluation, and (2) no account is taken for either favorable or antagonistic correlations among traits.

We have noticed that some traits are given a high selection emphasis, mainly due to a high economic value. For example, in the New Zealand sheep maternal breeding objective, lamb survival accounts for $13 \%$ of the index percent emphasis, which is higher than the percent emphasis calculated for wool or adult size. In reality, the selection pressure on lamb survival is low and consequently weak. Survival traits have low heritability and are evaluated with very low accuracy in young ram selection candidates compared with other traits. Thus, it is unlikely that strong selection pressure exists; therefore, it is difficult to realize any genetic improvement. This implies that the current selection index weight percent emphasis approach creates a significant issue in the reporting of indexes; for example, industry stakeholders can be fooled into thinking that traits important to them are being addressed by the index, when, in reality, they are not.

To alleviate the over- and underestimation of traits that have extreme economic values, a simple correction is to account for the evaluation accuracy. For example, the low accuracy for survival traits could shrink their emphasis to some degree. However, this still does not address the trait genetic correlations. In an index where some traits are highly correlated, the summation of their genetic SD is an underestimation of the total index SD; therefore the proportion of each trait's SD in the current selection emphasis overestimates the antagonistically correlated traits, and underestimates the favorably correlated traits.

Cunningham and Tauebert (2009) proposed a method for defining the relative importance of traits in an index that used covariances between targeted traits and measured variates. They demonstrated that ignoring correlated traits in a dairy breeding objective led to overestimation of production trait emphasis. However, with their method, when 2 selection criteria are both influential and also highly correlated, dropping either may be shown to have minimal effect, whereas if both were dropped simultaneously, the impact could be substantial. Further limitations of their method are that the traits with unfavorable responses to selection also have negative relative emphasis, and trait emphases do not sum up to $100 \%$.

Here we propose a set of new emphasis methods adjusted by either or both accuracy and genetic correlation. In short, we weight the SD of each trait by that trait's average accuracy across all selection candidates, then calculate subindex emphasis within each genetically highly correlated subgroup, and weight them by their group total emphasis.

\section{MATERIALS AND METHODS}

The aim of this study was to compare our new methods with existing methods in calculating selection emphasis. We first outline our new methods as an extension of the conventional approach with account for EBV accuracies and trait correlations. We then describe clustering methods that define subindexes with high within- and low between-subindex trait correlations. These subindex definitions are used to help account for any potential distortions created by trait correlations. We then describe how the new methodology can be deployed when sets of EBV and economic weights are available on selection candidates, as opposed to having accurate knowledge of the genetic variance or covariance matrix. We conclude with the description of a small meta-analysis study to show the differences between method outcomes across 8 different indexes.

\section{Selection Index Emphasis Methods}

The formula to calculate a linear selection index $(I)$ for a selection candidate is as follows:

$$
I=\sum_{i=1}^{t} a_{i} g_{i}
$$


where $t$ is the number of traits, and $a_{i}$ and $g_{i}$ are the economic weight and genetic merit of the $i$ th trait, respectively. The term $g_{i}$ could be replaced by EBV when it is calculated from a multitrait BLUP model. This can be rearranged to the following form:

$$
I=\sum_{i=1}^{t} a_{i} g_{i}=\sum_{i=1}^{t} a_{i} \sigma_{g_{i}} \frac{g_{i}}{\sigma_{g_{i}}}
$$

where $\sigma_{g_{i}}$ is the genetic SD of the ith trait, and $a_{i} \sigma_{g_{i}}$ is the weight given to the standardized EBV of trait $i$, $\frac{g_{i}}{\sigma_{g_{i}}}$. Traditionally, the selection emphasis formula is calculated as the absolute value of the standardized weight of trait $i$ as a percentage of summed weights of all traits, assuming all targeted traits are measured variates:

$$
e 1_{i}=\frac{\left|a_{i}\right| \sigma_{g_{i}}}{\sum_{i=1}^{t}\left|a_{i}\right| \sigma_{g_{i}}} \times 100 \%=\left|a_{i}\right| \sigma_{g_{i}}\left[\mathrm{a}^{\prime} \mathrm{I} \sigma_{\mathrm{g}}^{2} \mathrm{a}\right]^{-\frac{1}{2}} \times 100 \%
$$

where I is an identity matrix of rank $t, a$ is the vector of absolute values of economic values with transpose $\mathrm{a}^{\prime}$, and $I \sigma_{\mathrm{g}}^{2}$ is a diagonal matrix with elements that are the genetic variance of each trait. The second half of the equation is a matrix notation equivalent to the first half.

It is easy to observe from Equation [1] that traits with higher economic value or genetic SD have higher selection emphasis. To further adjust the selection emphasis by genetic evaluation accuracy, we can multiply each trait's genetic SD in Equation [1] by the expected accuracy of prediction in selection candidates, $\rho$, as in $\sigma_{g}^{*}=\sigma_{g} \times \rho$, so that

$$
e 2_{i}=\left|a_{i}\right| \rho_{i} \sigma_{g_{i}}\left[\mathrm{a}^{\prime} \mathrm{I}\left(\sigma_{\mathrm{g}} \circ \rho\right)^{2} \mathrm{a}\right]^{-\frac{1}{2}} \times 100 \%,
$$

where $\rho_{i}$ is the average accuracy of all selection candidates for trait $i, \rho$ is the vector of accuracy, and $\circ$ is the Hadamard product (element-wise product) sign. This gives greater emphasis to traits with higher accuracies. The summation of both $e 1_{i}$ and $e 2_{i}$ over all traits is $100 \%$.

It is also easy to observe from Equation [1] that the identity matrix form does not account for the genetic correlations among traits. To address this, we cluster the index into subindexes that each contain closely related traits. The weight $(w)$ for the $k$ th subindex of closely related traits is

$$
w_{k}^{\prime}=\left[\mathrm{a}_{\mathrm{k}}^{\prime} \mathbf{G}_{\mathrm{kk}} \mathrm{a}_{\mathrm{k}}\right]^{\frac{1}{2}},
$$

where $a_{\mathrm{k}}$ is a subset of a, and $\mathbf{G}_{\mathrm{kk}}$ is the $k$ th subset of G. To ensure the subindex weights sum up to $100 \%, w_{k}$ can be converted to percentage:

$$
w_{k}=\frac{w_{k}^{\prime}}{\sum_{k=1}^{m} w_{k}^{\prime}}=\frac{\left[\mathrm{a}_{\mathrm{k}}^{\prime} \mathbf{G}_{\mathrm{kk}} \mathrm{a}_{\mathrm{k}}\right]^{\frac{1}{2}}}{\sum_{k=1}^{m}\left[\mathrm{a}_{\mathrm{k}}^{\prime} \mathbf{G}_{\mathrm{kk}} \mathrm{a}_{\mathrm{k}}\right]^{\frac{1}{2}}}
$$

where $m$ is the total number of subindex groups. Note that the denominator is not equivalent to $\left[\mathrm{a}^{\prime} \mathbf{G a}\right]^{\frac{1}{2}}$, because the between-subindex grouping covariances are assumed to be zero in our formulation. Combining Equations [1] and [3] or [2] and [3], with and without consideration for differences in trait accuracy respectively, we get 2 additional subindex weighted new emphasis equations:

$$
e 3_{i k}=e 1_{i k} w_{k} \times 100 \%=\left|a_{i}\right| \sigma_{g_{i}}\left[\mathrm{a}^{\prime} \mathrm{I} \sigma_{\mathrm{g}}^{2} \mathrm{a}\right]^{-\frac{1}{2}} w_{k} \times 100 \%
$$

and

$e 4_{i k}=e 2_{i k} w_{k} \times 100 \%=\left|a_{i}\right| \rho_{i} \sigma_{g_{i}}\left[\mathrm{a}^{\prime} \mathrm{I}\left(\sigma_{\mathrm{g}} \circ \rho\right)^{2} \mathrm{a}\right]^{-\frac{1}{2}} w_{k} \times 100 \%$.

The summation of either $e 3_{i}$ or $e 4_{i}$ over all traits is $100 \%$. Matrices $\mathbf{G}$ and $\mathbf{G}_{\mathrm{kk}}$ can be adjusted by average trait accuracy, as $\mathbf{G}^{*}=\mathbf{G} \circ\left(\rho \rho^{\prime}\right)$ and $\mathbf{G}_{\mathrm{kk}}^{*}=\mathbf{G}_{\mathrm{kk}} \circ\left(\rho \rho^{\prime}\right)$; thus

$$
w_{k}^{*}=\frac{\left[\mathrm{a}_{\mathrm{k}}^{\prime} \mathbf{G}_{\mathrm{kk}}^{*} \mathrm{a}_{\mathrm{k}}\right]^{\frac{1}{2}}}{\sum_{k=1}^{m}\left[\mathrm{a}_{\mathrm{k}}^{\prime} \mathbf{G}_{\mathrm{kk}}^{*} \mathrm{a}_{\mathrm{k}}\right]^{\frac{1}{2}}} \text {. }
$$

Because the trait variance is now smaller, variance of $w_{k}^{*}$ is also smaller than $w_{k}$. Also,

$$
e 3_{i k}^{*}=e 1_{i k} w_{k}^{*} \times 100 \%=\left|a_{i}\right| \sigma_{u_{i}}\left[\mathrm{a}^{\prime} \mathrm{I} \sigma_{\mathrm{g}}^{2} \mathrm{a}\right]^{-\frac{1}{2}} w_{k}^{*} \times 100 \%
$$




$$
e 4_{i k}^{*}=e 2_{i k} w_{k}^{*} \times 100 \%=\left|a_{i}\right| \rho_{i} \sigma_{u_{i}}\left[\mathrm{a}^{\prime} \mathrm{I} \sigma_{\mathrm{g}}^{2} \mathrm{a}\right]^{-\frac{1}{2}} w_{k}^{*} \times 100 \% .
$$

\section{Clustering Methods}

The genetic correlation matrix $(\mathbf{C})$ is a form of affinity matrix, with each element measuring the relationship between a pair of traits. It is symmetric, semidefinite positive, and convex, with ellipsoidal geometry (Rousseeuw and Molenberghs, 1994). Common clustering methods such as k-means or hierarchical and Gaussian mixture clustering are suitable for this type of affinity matrix. Because in most cases the number of clusters is unknown, we decided to use hierarchical clustering in this study. We implemented hierarchical clustering with the ward.D2 agglomeration method in R software (https://www.r-project.org/) stats package hclust function (Ward, 1963; Murtagh and Legendre, 2014) operating on a dissimilarity matrix of $\mathbf{D}=1-$ $\left|\mathbf{C}^{*}\right|$, where $\mathbf{C}^{*}$ is accuracy-adjusted $\mathbf{C}$, as

$$
\mathbf{C}^{*}=\mathbf{C} \circ\left(\rho \rho^{\prime}\right),
$$

where $\left|\mathbf{C}^{*}\right|$ is the similarity matrix. We take absolute values of correlation coefficients within $\mathbf{C}^{*}$ because the directions of trait correlations do not matter in the scale of similarity.

The criterion ward.D2 agglomeration method minimizes the sum of squares of Euclidean distances within a cluster (Murtagh and Legendre, 2014), which is also the height of the dendrogram for each merging step.

The difference in the height of the dendrogram between $i$ th and $(i-1)$ th merging step was used to determine the optimal number of clusters. The merging step with the largest change in height from its previous step was considered the optimal cut point. In the situation where the changes in height from all merges are close, the user could define their ideal number of clusters, and the hierarchy tree would then be cut according to the number of clusters specified by the user, instead of automatically based on changes in height.

\section{Alternative Deployment Using Estimated Breeding Value}

In some situations, the genetic (co)variance components, $\mathbf{G}$, are not available. It can be convenient to construct $\mathbf{G}$ and $\mathbf{C}$ as (co)variances and correlations among EBV for a relevant set of selection candidates. In this situation, the total EBV variances are smaller than the original genetic variance, as breeding value predictions have been regressed toward genetic group average according to their accuracy of prediction. Thus, $\mathbf{G}$ and $\mathbf{C}$ obtained from analysis of sets of EBV do not need to be adjusted by $\rho$, as by using $\mathbf{G}$ and $\mathbf{C}$ instead of $\mathbf{G}^{*}$ and $\mathbf{C}^{*}$, and using Equations [5] and [6] instead of [7] and [8] to calculate selection emphasis, because the effects of differences in accuracy across traits are already accounted for in the EBV (co)variances. Note that the adjusted $\mathbf{G}^{*}$ and $\mathbf{C}^{*}$ are not identical to $\mathbf{G}$ and $\mathbf{C}$ computed by EBV, because the average trait accuracy instead of the individual animal accuracy was used.

\section{Materials}

Three data sets were first used to illustrate the methods (Table 1). The first data set is the New Zealand sheep maternal selection index as formulated in 2015 (Santos et al., 2015). This index included 4 growth traits: weaning weight (WWT), carcass weight (CWT), ewe mature weight (EweWT), and maternal weaning weight (WWTmat); 3 fleece weight traits: hogget fleece weight (HFW), lamb fleece weight (LFW), and ewe fleece weight (EFW); 3 fecal egg count traits: FEC1, FEC2, and adult fecal egg count (AFEC); and 2 survival traits: number of lambs born (NLB) and direct and maternal survival count (SUR and SURmat, respectively). The trait SD of genetic effects and accuracy per trait as an average of individual genetic evaluation accuracies were from young ram selection candidates (Santos et al., 2015). The genetic correlation matrix is shown in Table 2.

The second data set is the New Zealand dairy breeding objective for 2020, shown in Table 1 (DairyNZ, 2020) and the genetic correlation matrix using pedigree analysis-based EBV of progeny-tested crossbred breed selection candidates evaluated in 2019, shown in Table 3. Each selection candidate had their own trait accuracy contributing to the genetic (co)variances and correlations. The trait average accuracies across all animals could potentially be used for the accuracy-adjusted emphasis methods, except that here EBV is already an accuracy-adjusted genetic value. This included 8 traits in total: milk fat (FAT), milk volume (VOL), milk protein (PROT), live weight (LW), residual survival (RS), SCS, fertility (FER), and BCS.

The third data set is the Irish Cattle Breeding Federation (ICBF) dairy economic breeding index (EBI) in 2020 (Table 1; Ross Evans, ICBF, personal communication) and the genetic correlation matrix based on genetic EBV (GEBV) of active bulls with reliability above $50 \%$ (ICBF, 2020). As with the previous data set, accuracy information is incorporated by use 
Table 1. The 2015 New Zealand sheep maternal selection index, dairy cattle breeding objective, and Irish Cattle Breeding Federation (ICBF) dairy economic breeding index and genetic evaluation

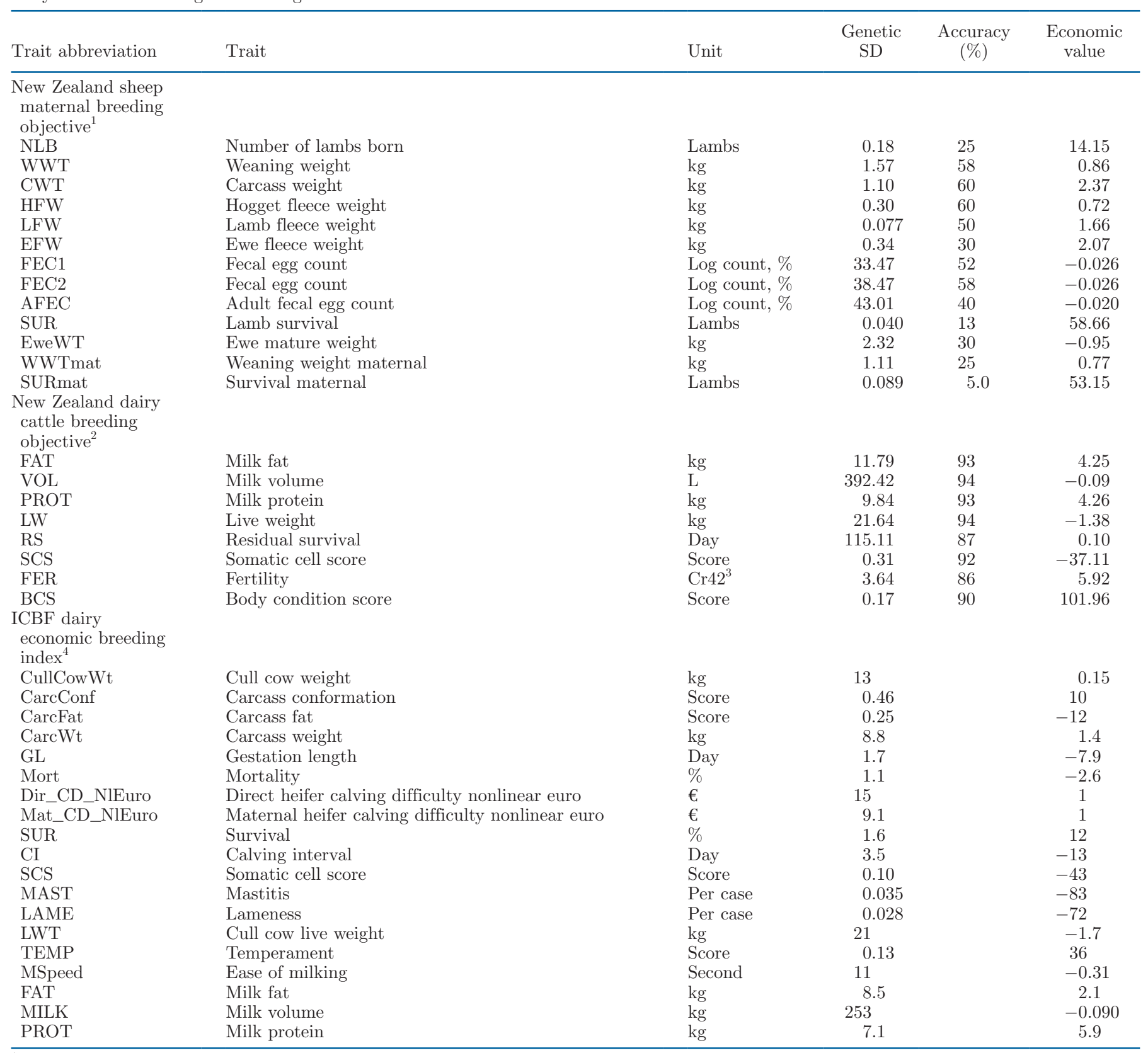

${ }^{1}$ Santos et al. (2015). Economic values are in New Zealand cent.

${ }^{2}$ DairyNZ (2020). Economic values are in New Zealand dollar.

${ }^{3} \mathrm{Cr} 42$ = calving rate in the first $42 \mathrm{~d}$ after planned start of calving in lactation 2,3 , and 4 (https://www.dairynz.co.nz/news/genetics-and -liveweight-gain-influence-heifer-puberty-timing/).

${ }^{4}$ Ross Evans, ICBF, Bandon, personal communication. No accuracy information is available. Economic values are in euro.

of EBV (co)variances. The index included 21 traits: cow weight at culling (CullCowWt), carcass conformation (CarcConf), carcass fat (CarcFat), carcass weight (CarWt), gestation length (GL), mortality (MORT), direct and maternal calving difficulty nonlinear euro (Dir_CD_NlEuro, Mat_CD_NlEuro), survival (SUR), calving interval (CI), SCS, mastitis (MAST), lameness (LAME), cull cow live weight (LWT), temperament (TMP), ease of milking (MSpeed), and milk fat (FAT), volume (MILK), and protein (PROT).

In all 3 data sets we chose to use progeny-tested males, but in practice many other categories of selec- 


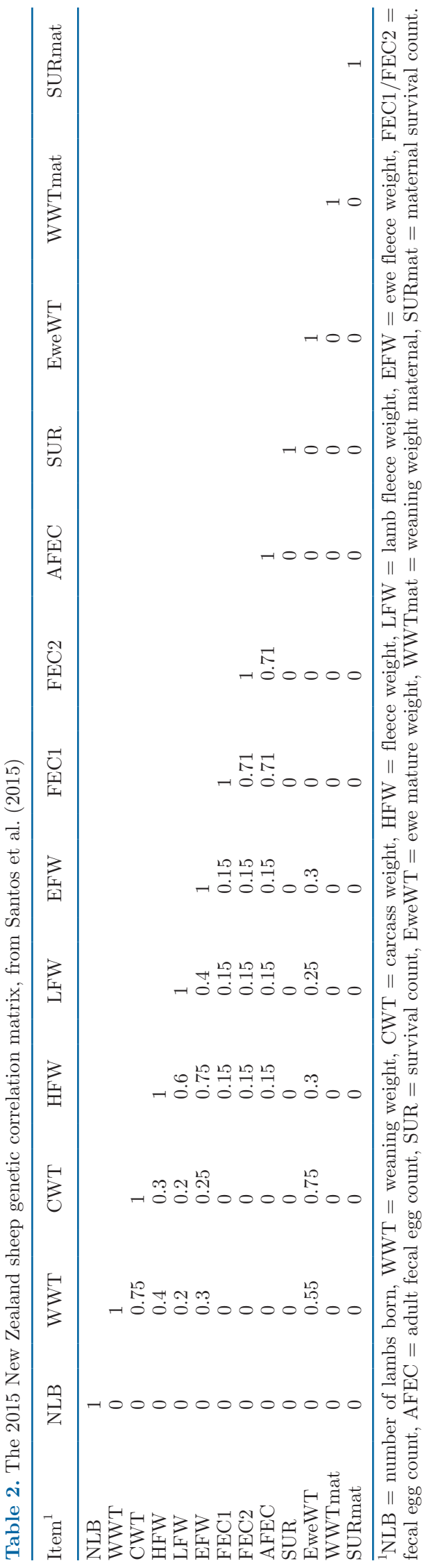

tion candidates could be evaluated using the same methods. For example, selection of dams of sons can be an important driver of genetic gain. Therefore, applying this method to these dams would be of additional interest. In most livestock industries, selection of males contributes more to genetic progress than selection of females; however, the case of genomic selection of potential bull-dams in dairy does introduce a novel category of selection candidates that could be evaluated.

\section{RESULTS}

\section{Summary Statistics of Example Selection Index Data Sets}

The average accuracy of genetic evaluation in the New Zealand sheep selection index was $0.39 \pm 0.18$, and the mean absolute value of the off-diagonal trait genetic correlation matrix was $0.13 \pm 0.22$. After adjusting the genetic correlation matrix by accuracy, as shown in Equation [9], the mean genetic correlation has become $0.03 \pm 0.06$. The average accuracy of genetic evaluation in New Zealand dairy cattle selection index was $0.91 \pm 0.03$, and the mean absolute value of the off-diagonal trait correlation matrix using selection candidates' EBV was $0.29 \pm 0.21$. For ICBF dairy EBI, the mean absolute value of the off-diagonal trait correlation matrix using GEBV was $0.21 \pm 0.18$. In short, the New Zealand sheep selection index had a lower average genetic trait accuracy, higher accuracy variation, and sparser genetic correlation matrix compared with both New Zealand and Ireland dairy selection indexes.

\section{Clustering and Emphasis of Example Selection Index Data Sets}

The clustering results and the rearranged genetic correlation matrix adjusted by accuracy for the New Zealand sheep data set are shown in Figure 1. The hierarchical clustering with our optimal cut point algorithm generated 4 groups. It can be clearly seen that in the rearranged $\mathbf{C}$ matrix adjusted by accuracy, the traits with higher correlations are grouped together, and correlations between groups are much lower $(\leq 0.1)$ than within groups, except for group 1: these are the traits that have 0 correlations to the rest of the selection index, due to either low heritability (maternal traits) or low variation (survival count traits), whereas traits within groups 2,3 , and 4 are traits with similar biological function.

The traditional selection emphasis method gave lots of emphasis to group 1 traits but also group 2 traits (Figure 2). By adjusting these with accuracy, the emphases for group 1 traits were slightly reduced 
Table 3. The 2020 New Zealand dairy cattle correlation matrix of crossbred EBV ${ }^{1}$

\begin{tabular}{|c|c|c|c|c|c|c|c|c|}
\hline Item $^{2}$ & FAT & VOL & PROT & LW & $\mathrm{RS}$ & SCS & FER & BCS \\
\hline VOL & 0.36 & 1 & & & & & & \\
\hline PROT & 0.45 & 0.95 & 1 & & & & & \\
\hline RS & 0.025 & -0.045 & -0.029 & 0.19 & 1 & & & \\
\hline SCS & 0.38 & 0.31 & 0.47 & 0.14 & -0.30 & 1 & & \\
\hline FER & -0.021 & -0.20 & -0.21 & -0.18 & -0.094 & -0.32 & 1 & \\
\hline
\end{tabular}

${ }^{1}$ DairyNZ (2020).

${ }^{2} \mathrm{FAT}=$ milk fat, $\mathrm{VOL}=$ milk volume, $\mathrm{PROT}=$ milk protein, $\mathrm{LW}=$ live weight, $\mathrm{RS}=$ residual survival, $\mathrm{SCS}=$ somatic cell score, $\mathrm{FER}=$ fertility, BCS = body condition score.

(absolute changes were $-0.72 \%$ for WWTmat, $-6.56 \%$ for SUR, $-2.16 \%$ for NLB, and $-19.45 \%$ for SURmat), and the scales of reduction were proportional to the difference of their accuracies from the mean accuracy of all traits. Similarly, traits with relatively high accuracies in groups 2 and 4 were slightly higher (absolute changes were $6.09 \%$ for WWT, $-0.1 \%$ for EweWT, $12.51 \%$ for CWT, $1.34 \%$ for AFEC, $3.09 \%$ for FEC1, and $4.56 \%$ for FEC2). The CWT trait increased the most in emphasis, reflecting that it had the highest accuracy among all traits and a combination of relatively

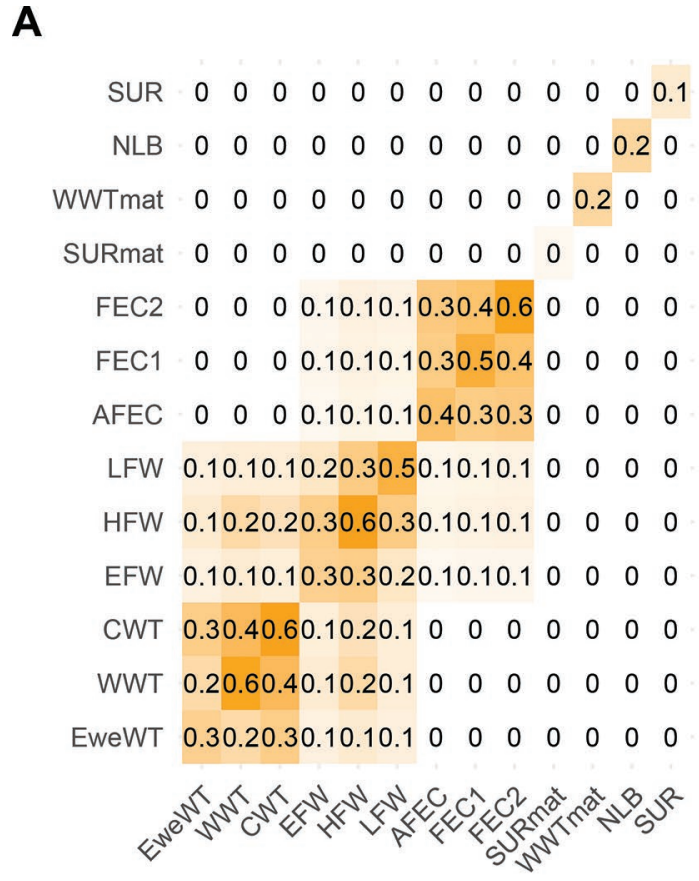

high genetic SD and absolute economic value. In Santos et al. (2015), EweWT was given a negative relative emphasis $(-19.3 \%)$ based on the response to the selection method proposed by Cunningham and Tauebert (2009), whereas our subindex and accuracy-weighted method gave $9.44 \%$ with all trait emphases adding up to $100 \%$. By subindex grouping or when clustering traits together, group 2 attained the highest group emphasis (43\%, Figure 2, compared with $30 \%$ with the traditional method), whereas group 1 emphasis shrunk the most $(19 \%$, compared with $51 \%$ with the tradi-

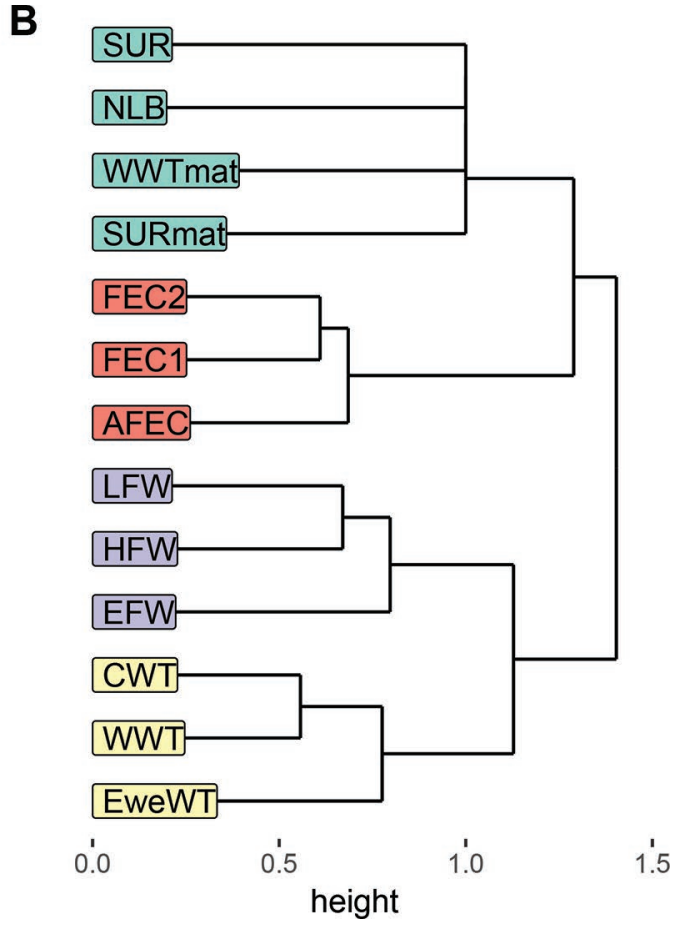

Figure 1. Hierarchical clustering results based on genetic relationship matrix of 2015 New Zealand sheep. (A) Genetic relationship matrix heatmap rearranged by clusters. The colors gradually change from white to orange as the absolute correlations go from 0 to 1 . (B) Dendrogram based on hierarchical clustering. The height is the ward.D2 agglomeration criterion between a pair of nodes. Traits in the same clusters are labeled in the same color. Trait names: WWT $=$ weaning weight, WWTmat $=$ weaning weight maternal, CWT $=$ carcass weight, EweWT $=$ ewe mature weight, $\mathrm{HFW}=$ fleece weight, $\mathrm{LFW}=$ lamb fleece weight, $\mathrm{EFW}=$ ewe fleece weight, FEC1/FEC2 = fecal egg count, AFEC = adult fecal egg count, NLB = number of lambs born, SUR = survival count, SURmat = maternal survival count. 
tional method). Groups 3 and 4 also increased in their group emphases with the subindex grouping method compared with the traditional method. Consequently, the subindex grouping method increased all the singletrait emphases in groups 2,3 , and 4 and reduced those in group 1. When both group weight and accuracy were used to adjust the selection emphases, the total group weight of each group stayed the same, but the emphasis of each trait within a group were reranked by their accuracy.

In the New Zealand dairy cattle data set, the correlations between groups were higher than those in the New Zealand sheep data set (Figure 3). Most traits had an absolute correlation lower than 0.4 with traits in other groups. For example, the range of absolute within-group correlations and the range of absolute betweengroup correlations for group 1 had overlap (0.4-0.9 vs. 0-0.5). Here PROT, VOL, and LW were in group 1, BCS and FER were in group 4, and SCS and RS were by themselves in separate groups. The traditional emphases were similar to the official emphases using all breeds (Figure 4, DairyNZ). We did not use the accuracy-adjusted method here, because this data set used GEBV to calculate the $\mathbf{G}$ and $\mathbf{C}$ matrices. Results showed that group 2 total absolute emphasis shrank from $49 \%$ by traditional method to $23 \%$ by subindex weighted method, due to the positive correlations among all traits ( 0.53 between LW and VOL and 0.57 between LW and PROT), but negative economic weight of LW (-1.38 NZD $/ \mathrm{kg} \mathrm{LW})$, which made the subindex weight of group 2 before scaling by those of all groups, $\mathrm{w}_{2}^{\prime}$, smaller than the summation of their within-group

absolute index weight; that is, $\left[\mathrm{a}_{\mathrm{k}}^{\prime} \mathbf{G}_{\mathrm{kk}} \mathrm{a}_{\mathrm{k}}\right]^{\frac{1}{2}}<\left[\mathrm{a}^{\prime} \mathrm{I} \sigma_{\mathrm{g}}^{2} \mathrm{a}\right]^{\frac{1}{2}}$. A consequence of the reduced emphasis on LW was that the other 3 group emphases all increased to take values between $4.4 \%$ to $11.04 \%$.

The ICBF dairy EBI data set includes 21 traits, and the correlations were much more complicated than the other 2 data sets (Figure 5). The grouping based on the optimal cut point generated 3 groups, with groups 3,4 , and 6 on Figure 5 not split. We manually defined $k=6$, as this resulted in functionally close traits being grouped together. In contrast to groups 2 and 3, groups 1, 4, and 5 had much higher GEBV correlations within subindex groups relative to correlations between groups. Group 3 contained mostly weakly correlated traits and were the last to be merged onto the hierarchy tree. The clustering ended up grouping together functionally similar or mathematically similar traits (scores and counts). Groups 1 and 2 were mostly production traits, and their group weights were reduced after subindex weighting (from 20 to $15 \%$ and from
35 and $25 \%$, respectively; Figure 6), whereas groups 4,5 , and 6 were increased (from 25 to $37 \%$, from 2.7 to $4.1 \%$, and from 1.4 to $2.2 \%$, respectively). Group 3 barely changed (absolute emphasis change was $0.07 \%$ ). The reductions in emphases for group 1 and 2 traits were due to the unfavorable relationships within their subindexes. In group 1 PROT, FAT, and MILK were positively correlated, but MILK was given a negative economic weight of $-\$ 0.09$. In group 2, LW was positively correlated with CacrWt and CullCowWt, but its economic weight was negative, unlike the other 2 traits (Table 1 and 4).

An additional 5 data sets were also used to analyze the effect of subindex weighting and accuracy on the changes of the emphasis compared with the traditional method (results not shown). Their result patterns were similar to the 3 examples previously described.

\section{DISCUSSION}

A major phenomenon in livestock breeding over the past 3 decades has been the expansion of breeding objectives and selection criteria to include more traits (Miglior et al., 2005, 2017; Cole and VanRaden, 2018). This trend is continuing with exploration of genomics approaches to include novel traits that are not practical or cost effective to record in the wider population, but which can be recorded in specific training populations (Byrne and Amer, 2018). For the many gene stock buyers and breeding companies that rely on selection indexes to aid in their decision making, an accurate description of the indexes they are using is important.

The traditional method of characterizing the emphasis placed on traits within an index reflects more the opportunity for selection with unlimited accuracy and in highly interpretable percentage terms. Alternatively, the value of the variate approach described recently by Cunningham and Tauebert (2009) uses the principle of opportunity cost to evaluate the importance of selection criteria and acknowledges the presence of correlations among traits and the accuracy of their selection. This paper has addressed different limitations in these contrasting approaches. Adjusting the traditional percent emphasis calculation by trait genetic evaluation accuracy and accounting for trait genetic correlations using clustering provides a middle ground, capturing the relative strengths of both approaches.

In real breeding situations, most genetic gain in overall merit is realized from the index traits with a large amount of genetic variation, high economic weighting, and sufficiently accurate EBV. Animal breeders have been keen to show that concerns about historic narrow selection focus on a small number of product output traits have been addressed with the introduction of new 
A
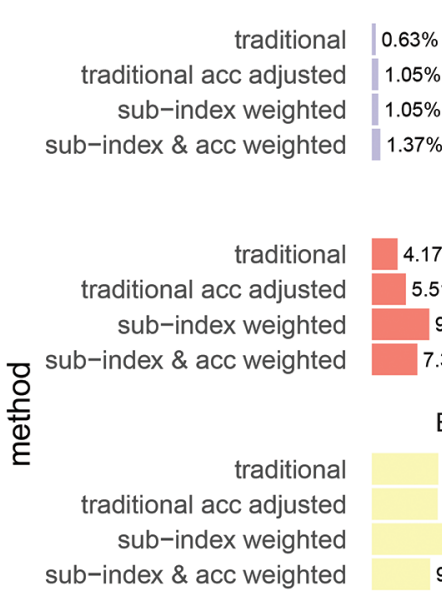

AFEC

$4.17 \%$

$5.51 \%$

$9.31 \%$

$7.37 \%$

EweWT

$10.77 \%$

$10.67 \%$

$9.44 \%$

SURmat

traditional acc adjusted

sub-index weighted

sub-index \& acc weighted

B

sub-index weighted

sub-index \& acc weighted

traditional

traditional acc adjusted

sub-index weighted

을 sub-index \& acc weighted

है

traditional acc adjusted

sub-index weighted

sub-index \& acc weighted

traditional

traditional acc adjusted

sub-index weighted

sub-index \& acc weighted

$3.85 \%$
$\quad 8.41 \%$
$3.17 \%$

$15.38 \%$

LFW
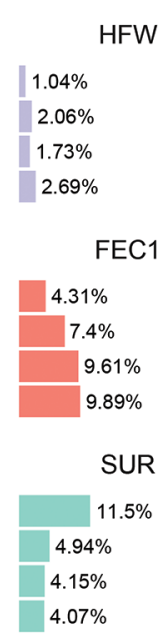

FEC1

HFW

$2.06 \%$

$1.73 \%$

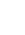

\section{C1}

$\%$



SUR

$1.5 \%$

$94 \%$

$\%$

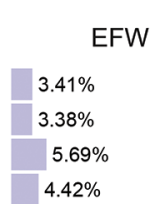

FEC2

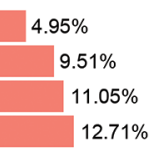

NLB

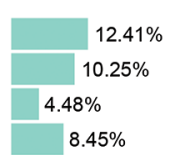

WWTmat

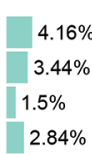

$1.5 \%$
$2.84 \%$

WWT

$6.62 \%$

$12.71 \%$

$9.45 \%$

$11.25 \%$

CWT

$12.74 \%$

$25.25 \%$

$18.2 \%$

$22.34 \%$

emphasis, \%
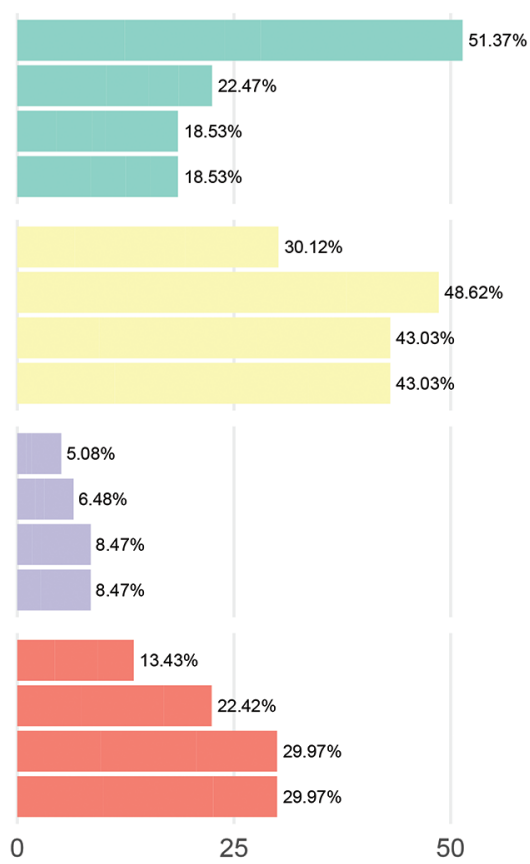

emphasis, \%

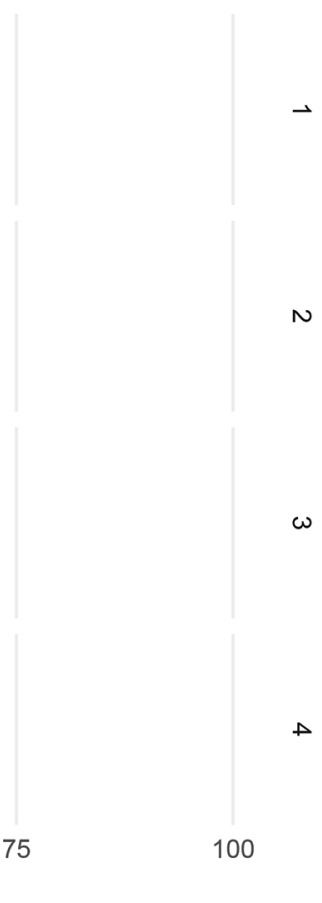

Figure 2. Selection emphasis $(e)$ percentages for 2015 New Zealand sheep by trait and method (A) and by group and method (B). Method: traditional $=e 1$, Equation [1]; subindex weighted $=e 3$, Equation [4]; traditional accuracy (acc)-adjusted $=e 2$, Equation [2]; subindex and acc weighted $=e 4$, Equation [5]. Trait names: WWT $=$ weaning weight, WWTmat $=$ weaning weight maternal, CWT $=$ carcass weight, EweWT $=$ ewe mature weight, $\mathrm{HFW}=$ fleece weight, $\mathrm{LFW}=$ lamb fleece weight, $\mathrm{EFW}=$ ewe fleece weight, $\mathrm{FEC} 1 / \mathrm{FEC} 2=$ fecal egg count, $\mathrm{AFEC}=$ adult fecal egg count, NLB = number of lambs born, SUR = survival count, SURmat = maternal survival count.

traits. Ignoring difference in accuracy of EBV in the methodology used to describe indexes has been shown here to be misleading in some situations. In our New
Zealand sheep selection index example, the traditional selection emphases calculated for low-heritability traits direct and maternal lamb survival were higher than 
A

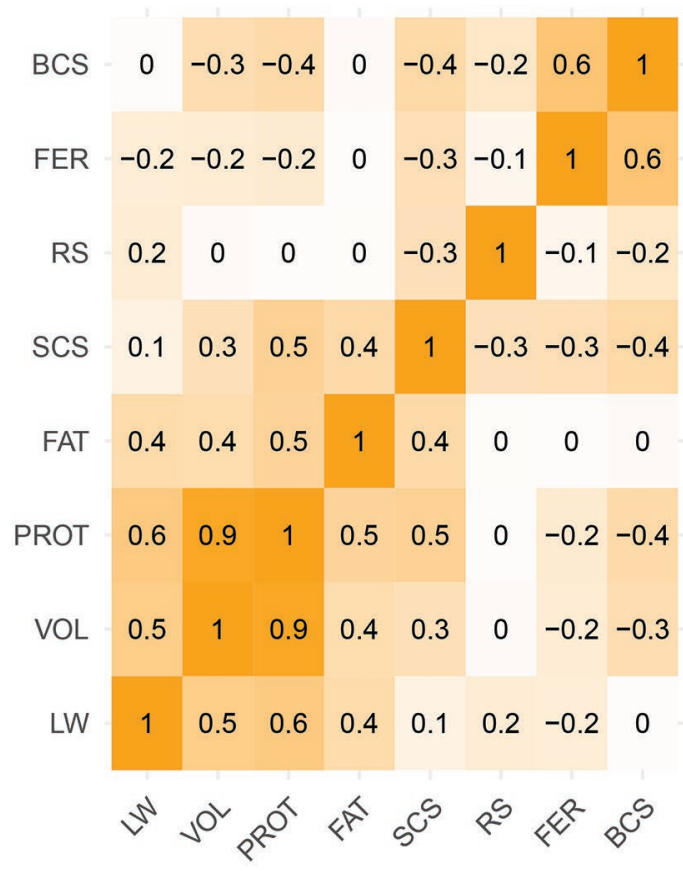

B

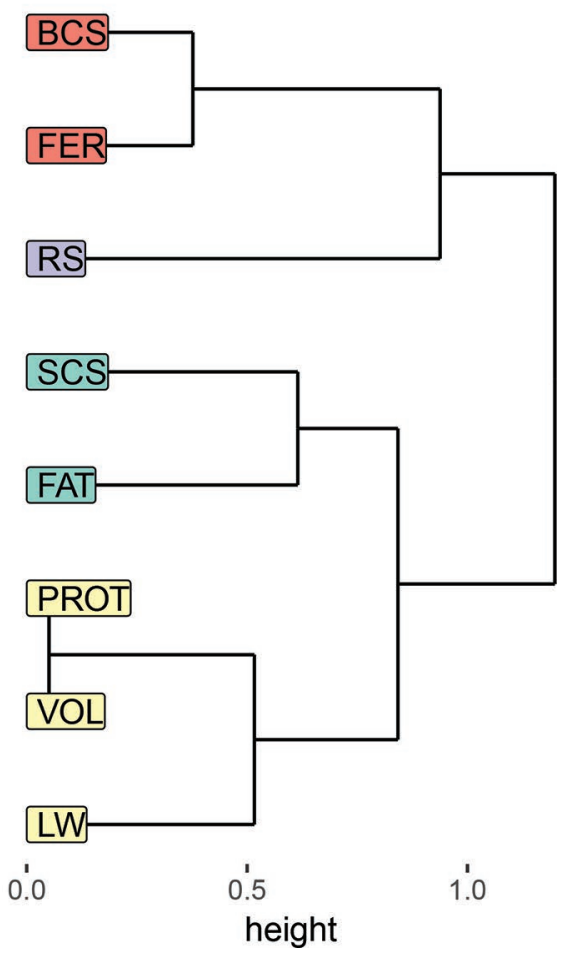

Figure 3. Hierarchical clustering results based on EBV correlation matrix of 2020 New Zealand dairy active bulls with reliability above $50 \%$. (A) Genetic relationship matrix heatmap rearranged by clusters. The colors gradually change from white to orange as the absolute correlations go from 0 to 1. (B) Dendrogram based on hierarchical clustering. The height is the ward.D2 agglomeration criterion between a pair of nodes. Trait names in the same clusters are labeled in the same color. Trait names: FAT $=$ milk fat, VOL $=$ milk volume, PROT $=$ milk protein, LW $=$ live weight, $\mathrm{RS}=$ residual survival, $\mathrm{SCS}=$ somatic cell score, $\mathrm{FER}=$ fertility, $\mathrm{BCS}=$ body condition score.

growth traits weaning weight and ewe weight. In reality, genetic trends in the New Zealand sheep industry have been dominated by genetic improvement in growth rate and litter size (Sise and Amer, 2009; Young, 2015). We believe that the most correct formulation for selection emphasis should be the one that reflects the actual selection pressure occurring in the breeding program.

The data sets used in this study have uniquely different characteristics. The New Zealand sheep data set represents some selection indexes that have highly correlated subsets of traits that have almost no correlation with other traits. It is easy to separate the overall index into subindex groups using any clustering method, and therefore this case study could make the most of the subindex weighting approach. Traits grouped together with neutral correlations behaved the same as when not grouped together. When indexes have many cross correlations among subsets of traits, related subindexes will either gain or lose the amount of emphasis assigned to them, depending on the strength and favorability in aggregate of their correlations. Stronger correlations contribute more covariance into the combined group weight, which alters the percent emphasis of all traits in the group.
Another reason for the larger observed difference between the old and new emphasis methods in the New Zealand sheep data set compared with the 2 other dairy data sets is likely due to the larger variation of trait accuracies in sheep compared with dairy selection candidates. The mean accuracies of sheep traits in our study range from 5 to $60 \%$, with an SD of the means of $18 \%$, whereas the SD of mean trait accuracies in New Zealand dairy cattle data set was much smaller at $2.9 \%$. The trait accuracy of ICBF dairy data set is not available, but it is likely to have a smaller SD than the sheep data set, given that dairy bulls have more progeny through artificial insemination and, hence, higher trait accurcies. With genomic selection in large populations becoming more accessible, reliabilities are more consistent across traits now than previously with conventional selection based on progeny testing. This might reduce the importance of weighting the percent emphasis method by accuracy in the future.

The last difference in the 3 data sets lies in the source of the data: the 2 dairy data sets represent the situation when genetic or genomic evaluations were used to approximate the genetic (co)variance components, and the New Zealand sheep data set represents the situa- 
tion when (co)variance components are available but not the $\mathbf{G}$ or $\mathbf{C}$ matrices. Matrices $\mathbf{G}$ or $\mathbf{C}$ may not be available because (1) they are not publicly available or
(2) $\mathbf{G}$ or $\mathbf{C}$ come from some submodules such as singletrait models, or a subset of multitrait models (such as production traits evaluated separately from health
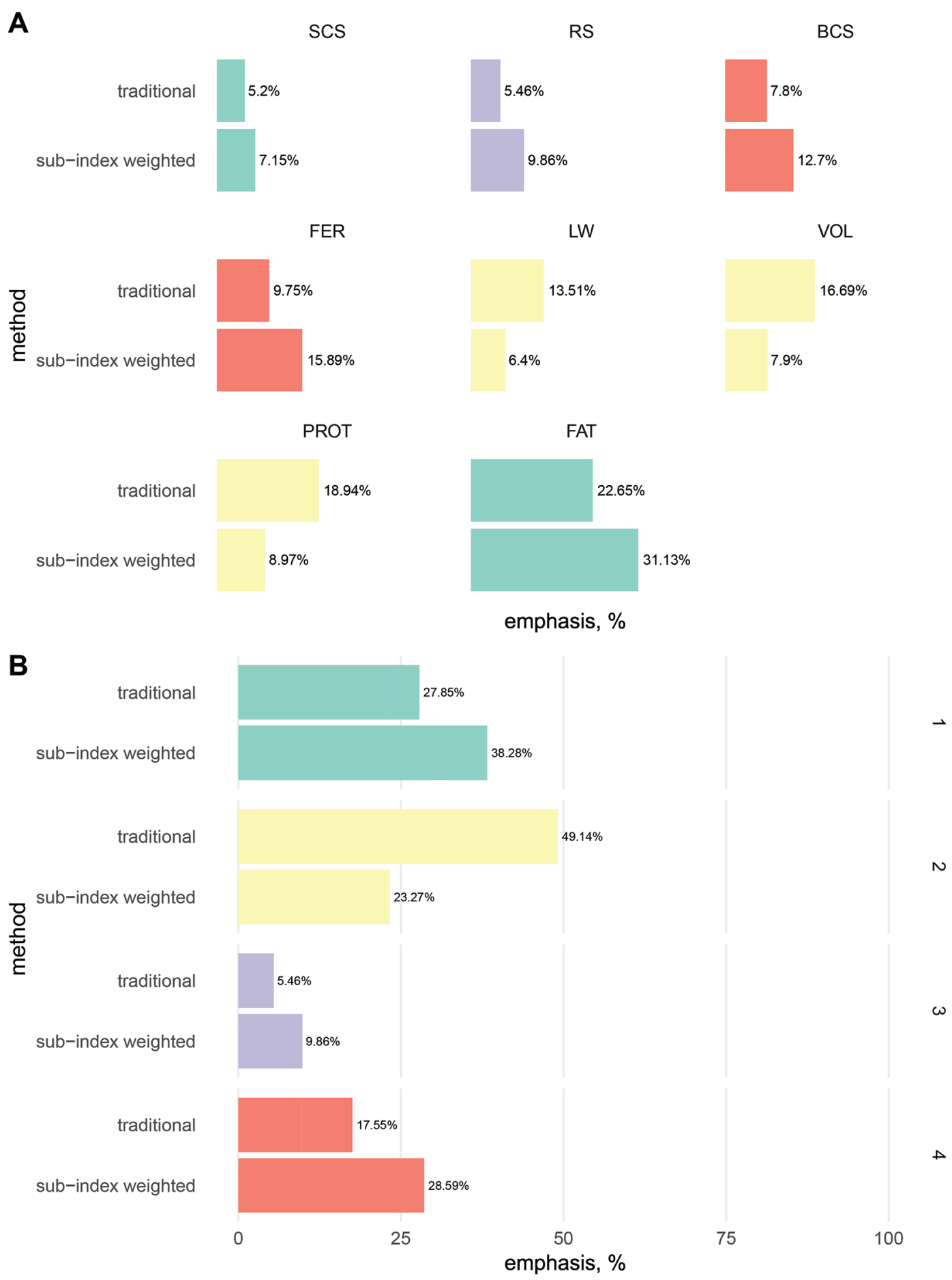

Figure 4. Selection emphasis (e) percentages for 2020 New Zealand dairy cattle by trait and method (A) and by group and method (B). Method: traditional $=e 1$, Equation [1]; subindex weighted $=e 3$, Equation [4]. In panel B, 1, 2, 3, and 4 are groups. Traits in the same clusters are labeled in the same color. Trait names: FAT $=$ milk fat, $\mathrm{VOL}=$ milk volume, $\mathrm{PROT}=$ milk protein, $\mathrm{LW}=$ live weight, $\mathrm{RS}=$ residual survival, $\mathrm{SCS}=$ somatic cell score, FER $=$ fertility, BCS = body condition score. 
and fertility traits for computational limits, especially the limitations created by genomics demands), but not from a full model.

\section{Limitations}

Our analysis of different index examples showed that for some of the selection indexes we investigated, the new methods generated similar emphases to those from the traditional method. Because of its simplicity, many industries may wish to persist with the current method, which is very straightforward to implement. Any distortions due to differences in accuracy or trait correlations may remain relatively constant within an industry over time, or between different breeding programs supporting similar industries. However, substantial efforts to improve the accuracy of breeding value prediction for secondary and novel traits in many industries will not result in higher apparent emphasis when the traditional method is used. These efforts must instead be shown through predictions of future or recent responses to selection, or both.
The new method we propose implicitly accounts for accuracy of breeding value prediction. This opens a new approach to defining the variation within, and correlation among, breeding objective traits when calculating the importance of component traits to an index. In some of our case studies, we used EBV to define trait variation, covariation, and absolute values of correlations. The advantage of this approach is that it can account for the dynamic effects of changes in breeding value accuracy, by comparing trait emphasis results for groups of selection candidates. Traits with nonlinear weightings in the index can also be considered (Berry et al., 2019). However, there are several potential limitations to be aware of. Any preselection of trait recording - for example, 2-stage selection approaches or culling based on early-life traits - could distort the variances and covariances observed. In this case, it might be preferable to evaluate percent emphasis based on the genetic (co)variance matrix, absolute value of economic weights, and trait accuracies, as in our example for the NZ sheep maternal index. Selection candidates should also be considered in a relatively tight time window, or
A

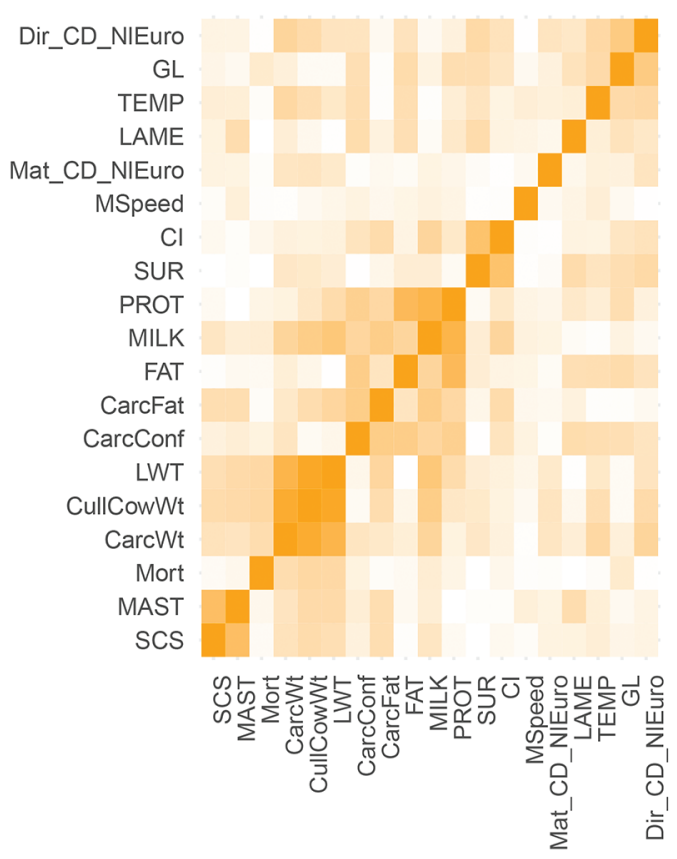

B

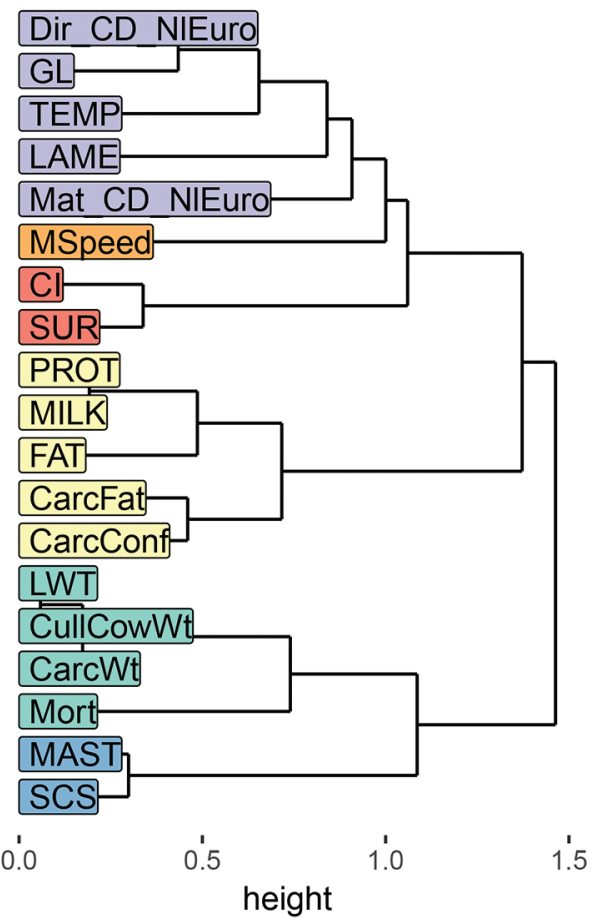

Figure 5. Hierarchical clustering results based on EBV correlation matrix of 2020 Irish Cattle Breeding Federation dairy economic breeding index. (A) Genetic relationship matrix heatmap rearranged by clusters. The colors gradually change from white to orange as the absolute correlations go from 0 to 1. (B) Dendrogram based on hierarchical clustering. The height is the ward.D2 agglomeration criterion between a pair of nodes. Trait names in the same clusters are labeled in the same color. Trait names: CullCowWt $=$ cow weight at culling, CarcConf $=$ carcass conformation, CarcFat $=$ carcass fat, CarcWt $=$ carcass weight, GL $=$ gestation length, Mort $=$ mortality, Dir_CD_NlEuro $=$ direct heifer calving difficulty nonlinear euro, Mat_CD_NlEuro = maternal heifer calving difficulty nonlinear euro, SUR = survival, CI = calving interval, SCS $=$ somatic cell score, MAST $=$ mastitis, LAME = lameness, LWT = cull cow live weight, TEMP $=$ temperament, MSpeed $=$ ease of milking, $\mathrm{FAT}=$ milk fat, MILK $=$ volume, $\mathrm{PROT}=$ protein. 
A

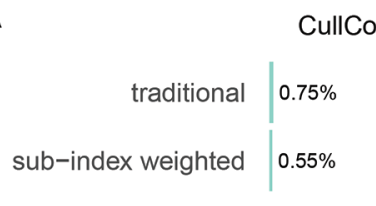

ஜ

sub-index weighted

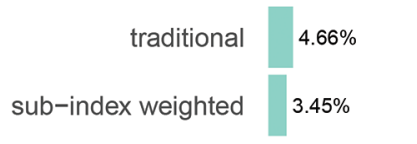

MILK

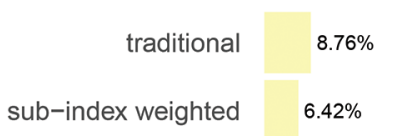

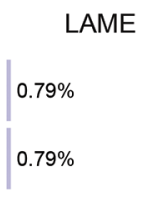

$\operatorname{SCS}$

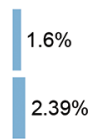

Mort

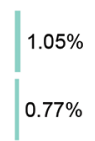

TEMP

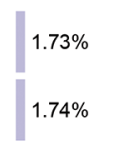

GL

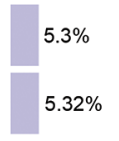

LWT

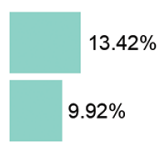

PROT
MAST

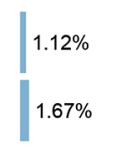

CarcConf

$1.84 \%$

$1.35 \%$
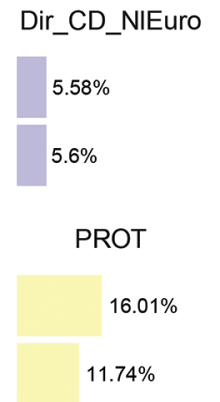

emphasis, \%

B

traditional

sub-index weighted

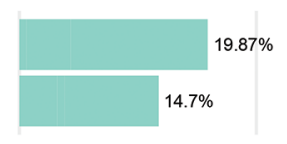

traditional

sub-index weighted

traditional

믈

sub-index weighted
traditiona

sub-index weighted

traditional
sub-index weighted

traditional
sub-index weighted
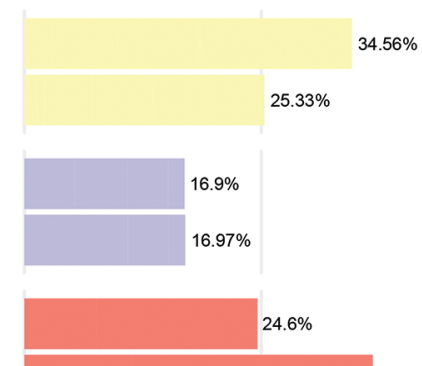

$4.6 \%$

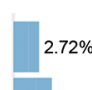

$4.06 \%$

traditional

sub-index weighted

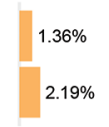

0

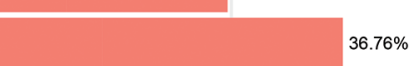

$36.76 \%$
FAT

$6.81 \%$

$4.99 \%$

Cl

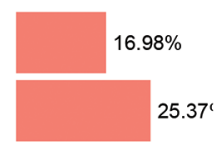

CarcFat

$1.14 \%$

$0.84 \%$

Mat_CD_NIEuro

$3.5 \%$

$3.51 \%$

SUR

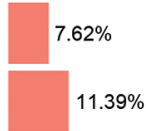

$11.39 \%$

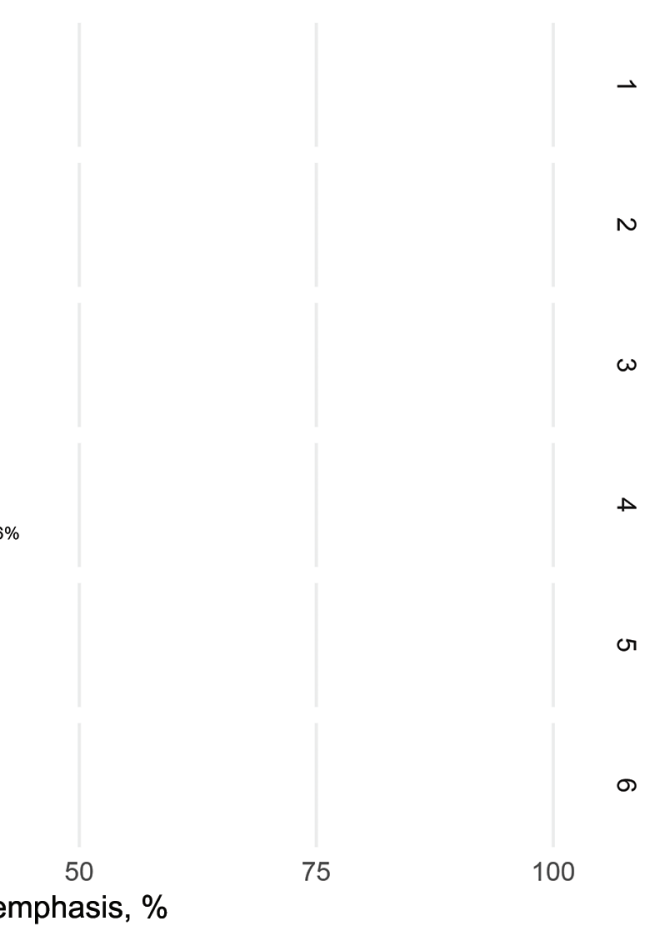

Figure 6. Selection emphasis (e) percentages for 2020 Irish Cattle Breeding Federation dairy economic breeding index by trait and method (A) and by group and method (B). Method: traditional =e1, Equation [1]; subindex weighted =e3, Equation [4]. In panel B, 1, 2, 3, and 4 are groups. Traits in the same clusters are labeled in the same color. Trait names: CullCowWt $=$ cow weight at culling, CarcConf $=$ carcass con-

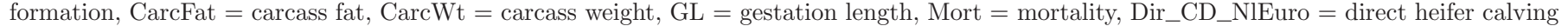

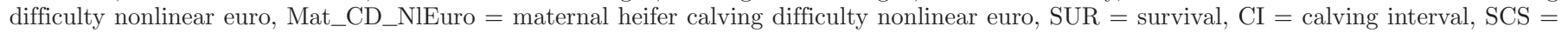
somatic cell score, MAST = mastitis, LAME = lameness, LWT = cull cow live weight, TEMP = temperament, MSpeed $=$ ease of milking, FAT $=$ milk fat, MILK $=$ volume, PROT $=$ protein . 


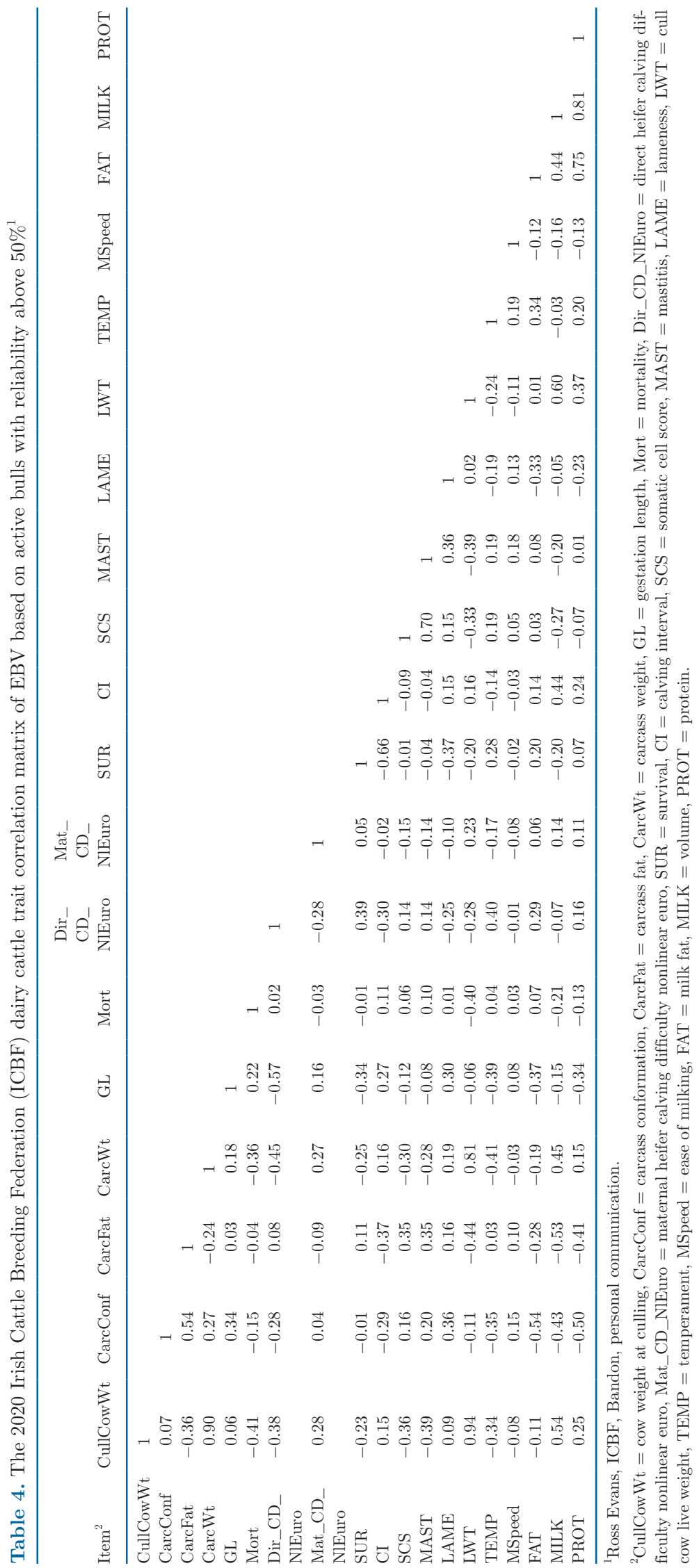


else genetic trend should be adjusted out of the variances for traits for which genetic trends are occurring. Where genetic trend is driven by more than one type of selection candidates that differ in their accuracy of breeding values (e.g., male selection candidates may be tested for more traits than female selection candidates, or both progeny-tested sires and young males with genomic predictions may jointly contribute to the pool of male candidates), it may be necessary to evaluate percent emphasis within candidate type and then aggregate across types according to the expected contribution to the parent pool and, consequently, to genetic trend.

\section{Clustering Versus Trait Function}

It is intuitive to think that traits with similar functions have higher correlations than those with different functions and, thus, that they should be clustered together. In the New Zealand dairy breeding objective example, the FAT trait was clustered with SCS rather than with other 2 milk traits, unlike in the ICBF dairy EBI example. Although it is true that in this example FAT had higher correlation with PROT than with SCS, the hierarchical clustering using the Ward.D2 agglomeration method started with 8 clusters of each trait by itself, then step by step joined the 2 closest clusters. In this case, PROT and VOL were joined first because their Ward.D2 distance was the closest (0.01). Next BCS and FER joined together at the second-closest distance (0.16), followed by LW merging into PROT and VOL at the distance of 0.30. Only then did SCS and FAT join, at the fourth-closest distance of 0.36 . The within-cluster to between-cluster variance ratio was also the highest in this way. The merge could be slightly different (1) if the $k$ changed, (2) using a different agglomeration method, or (3) using a different clustering method.

In many existing selection indexes, functionally close traits are categorized together into subindexes, the values of which were used for selection. To maintain this categorization while applying our subindex-weighted method of emphasis calculation, one can calculate trait emphasis using our method first and then add emphases of traits in the same functional subindex together to get their total emphases.

\section{CONCLUSIONS}

Our new method for defining the relevant importance of traits in a selection index generated more realistic results than traditional methods in some situations. The degree of change to traditional method depends on the value of within- and between-subindex trait correla- tions and consistency of genetic evaluation accuracy across all EBV. The new method is more complicated to deploy but opens up new and convenient deployment options, where predefined genetic (co)variance matrices are replaced by alternatives calculated from sets of EBV for defined groups of selection candidates.

\section{ACKNOWLEDGMENTS}

The authors acknowledge Bruno Santos (AbacusBio Ltd., New Zealand) for interpreting the data and helping with the analysis, and DairyNZ and ICBF for providing the genetic evaluations and consenting to the publication. Xinyue Zhang drafted the manuscript, analyzed the data, and designed the mathematical method; Peter Amer created the idea, designed the method, and revised the manuscript. Both authors approved the submitted version and agreed both to be personally accountable for their own contributions and to ensure that questions related to the accuracy or integrity of any part of the work are appropriately investigated, resolved, and the resolution documented in the literature. The authors have not stated any conflicts of interest.

\section{REFERENCES}

Berry, D. P., P. R. Amer, R. D. Evans, T. Byrne, A. R. Cromie, and F. Hely. 2019. A breeding index to rank beef bulls for use on dairy females to maximize profit. J. Dairy Sci. 102:10056-10072. https:/ /doi.org/10.3168/jds.2019-16912.

Byrne, T. J., and P. R. Amer. 2018. Breeding objectives in an era of transformational breeding technologies. Page 789 in Proc. World Congress on Genetics Applied to Livestock Production, Auckland, New Zealand. WCGALP.

Cole, J. B., and P. M. VanRaden. 2018. Symposium review: Possibilities in an age of genomics: The future of selection indices. J. Dairy Sci. 101:3686-3701. https://doi.org/10.3168/jds.2017-13335.

Cunningham, E. P., and H. Tauebert. 2009. Measuring the effect of change in selection indices. J. Dairy Sci. 92:6192-6196. https://doi .org/10.3168/jds.2007-565.

DairyNZ. 2020. Economic values. Accessed Mar. 17, 2020. https:// www.dairynz.co.nz/animal/animal-evaluation/interpreting-the -info/economic-values/.

Falconer, D., and T. Mackay. 1996. Introduction to Quantitative Genetics. 4th ed. Pearson Education India, Prentice Hall.

ICBF (Irish Cattle Breeding Federation). 2020. Understanding the Economic Breeding Index (EBI). Accessed Mar. 16, 2020. https:/ /www.icbf.com/wp/wp-content/uploads/2020/02/Understanding -EBI-PTA-BV-Spring-2020.pdf.

Manzanilla-Pech, C. I. V., R. F. Veerkamp, R. J. Tempelman, M. L. van Pelt, K. A. Weigel, M. VandeHaar, T. J. Lawlor, D. M. Spurlock, L. E. Armentano, C. R. Staples, M. Hanigan, and Y. de Haas. 2016. Genetic parameters between feed-intake-related traits and conformation in 2 separate dairy populations - The Netherlands and United States. J. Dairy Sci. 99:443-457. https://doi.org/10 $.3168 /$ jds.2015-9727.

Miglior, F., A. Fleming, F. Malchiodi, L. F. Brito, P. Martin, and C. F. Baes. 2017. A 100-Year Review: Identification and genetic selection of economically important traits in dairy cattle. J. Dairy Sci. 100:10251-10271. https://doi.org/10.3168/jds.2017-12968.

Miglior, F., B. L. Muir, and B. J. van Doormaal. 2005. Selection indices in Holstein cattle of various countries. J. Dairy Sci. 88:12551263. https://doi.org/10.3168/jds.S0022-0302(05)72792-2. 
Murtagh, F., and P. Legendre. 2014. Ward's hierarchical agglomerative clustering method: Which algorithms implement Ward's criterion? J. Classif. 31:274-295. https://doi.org/10.1007/s00357-014-9161-z.

Rousseeuw, P. J., and G. Molenberghs. 1994. The shape of correlation matrices. Am. Stat. 48:276-279. https://doi.org/10.1080/ 00031305.1994.10476079.

Santos, B. F. S., N. McHugh, T. J. Byrne, D. P. Berry, and P. R. Amer. 2015. Comparison of breeding objectives across countries with application to sheep indexes in New Zealand and Ireland. J. Anim. Breed. Genet. 132:144-154. https://doi.org/10.1111/jbg .12146 .

Sise, J. A., and P. R. Amer. 2009. SNP predictors to accelerate the rate of genetic progress in sheep. Pages 220-223 in Proc. Association for Advancement of Animal Breeding and Genetics, Barossa Valley. Accessed Apr. 28, 2020. http://www.sil.co.nz/.

VanRaden, P. 2002. Invited Review: Selection of dairy cattle for lifetime profit. Pages 127-130 in Proc. 7th World Congress on Genetics Applied to Livestock Production, Montpellier, France. Editions Quae.
VanRaden, P. M., J. B. Cole, and K. L. Parker Gaddis. 2018. Lifetime Net Merit 2018. AIPL Research Report NM\$7. Accessed Sep. 11, 2020. https://aipl.arsusda.gov/reference/nmcalc-2018.htm.

Ward, J. H. Jr. 1963. Hierarchical grouping to optimize an objective function. J. Am. Stat. Assoc. 58:236-244. https://doi.org/10 $.1080 / 01621459.1963 .10500845$.

William, N.S. 2016. A genetic and economic evaluation of lactose in the New Zealand dairy industry. PhD thesis, Animal Science. Massey University, Manuatu, New Zealand.

Young, M. 2015. The latest report card. Pages 104-108 in CountryWide, Oct. 2015. Accessed Apr. 28, 2020. https://www.blnzgenetics .com/files/1457587689420.pdf.

\section{ORCIDS}

X. Zhang () https://orcid.org/0000-0002-6439-2782

P. Amer (i) https://orcid.org/0000-0002-6428-7165 